\title{
PHOTOMETRIC AND INTERFEROMETRIC OBSERVATIONS OF A MID-LATITUDE STABLE AURORAL RED ARC
}

\author{
R. G. ROBLE, P. B. HAYS and A. F. NAGY \\ The University of Michigan, Ann Arbor, Mich. 48105, U.S.A.
}

(Received in final form 13 November 1969)

\begin{abstract}
Observational results of the mid-latitude stable auroral red arcs of October 30/31 and October 31/November 1, 1968 ar: presented. The measurements were made with a turret photometer and a 6 in. dia. high resolution, pressure scanning Fabry-Perot interferometer. The structure, intensity, and position of the red arc were determined from photometer scan measurements which show that the arc was stable, persisted for more than $12 \mathrm{hr}$, and moved southward during the night. The arc appeared to extend across the eastern part of the United States tilted to lines of constant magnetic $L$-shells. The results of the Doppler temperature measurements made with the Fabry-Perot interferometer show no measurable neutral gas temperature increase within the red arc.
\end{abstract}

\section{INTRODUCTION}

The mid-latitude stable auroral red arc was discovered by Barbier (1958) at times of geomagnetic disturbances during the last solar cycle maximum. The observed properties of the arcs, which occurred during the last solar cycle maximum, have been summarized by Roach and Roach (1963) and Marovich (1966). Hoch, Marovich and Clark (1968) and Ichakawa and Kim (1969) have reported on the reappearance of the stable auroral red arc for the current solar cycle maximum. Most of the observations made previously on the red arc have been photometric measurements. This paper summarizes the observed properties of the mid-latitude stable auroral red arcs of October 30/31 and October 31/ November 1, 1968 determined by a turret photometer and a high resolution Fabry-Perot interferometer. The measurements were made from the University of Michigan Airglow Observatory at Ann Arbor Michigan $\left(42^{\circ} 16^{\prime} \mathrm{N}, 83^{\circ} 44^{\prime} \mathrm{W}\right)$.

The turret photometer is of the type designed by Purdy, Mcgill and Roach (1961) and modified by Roble (1969); it was used to monitor the 5577 and $6300 \AA$ atomic oxygen emission lines and the 5400 and $6100 \AA$ continuum regions. In addition to the turret photometer a 6 in. dia., high resolution pressure scanning Fabry-Perot interferometer was used to measure the Doppler temperature of the $\left[O\left({ }^{1} D\right) \rightarrow O\left({ }^{3} P\right)\right] 6300 \AA$ atomic oxygen line. Both instruments have mirror scanning systems in order to scan in various sky positions. The details of the instruments are described elsewhere (Roble, 1969; Hays, Nagy and Roble, 1969); only the observational results are reported in this paper.

\section{THE STABLE AURORAL RED ARC OF OCTOBER 30/31, 1968}

A large geomagnetic storm, as revealed by the increased magnetic index, $K_{p}$, shown in Fig. 1, began at about 0000 UT on October 31, 1968, and both the Fabry-Perot interferometer and the airglow photometer were making nightglow measurements at that time. The early evening records gave some indication of an arc type enhancement at an approximate zenith angle of $30^{\circ}$ north of the geophysical observatory; however, it was not possible to resolve the feature completely because of some clouds in the area. No measurements were made from 0200 to 0545 UT because of cloudy conditions, but a general clearing occurred near $0600 \mathrm{UT}$ and a small $(100 \mathrm{R})$ red arc was observed from Ann Arbor at a zenith angle of approximately $50^{\circ}$ south in the meridian plane. The photometer mirror 


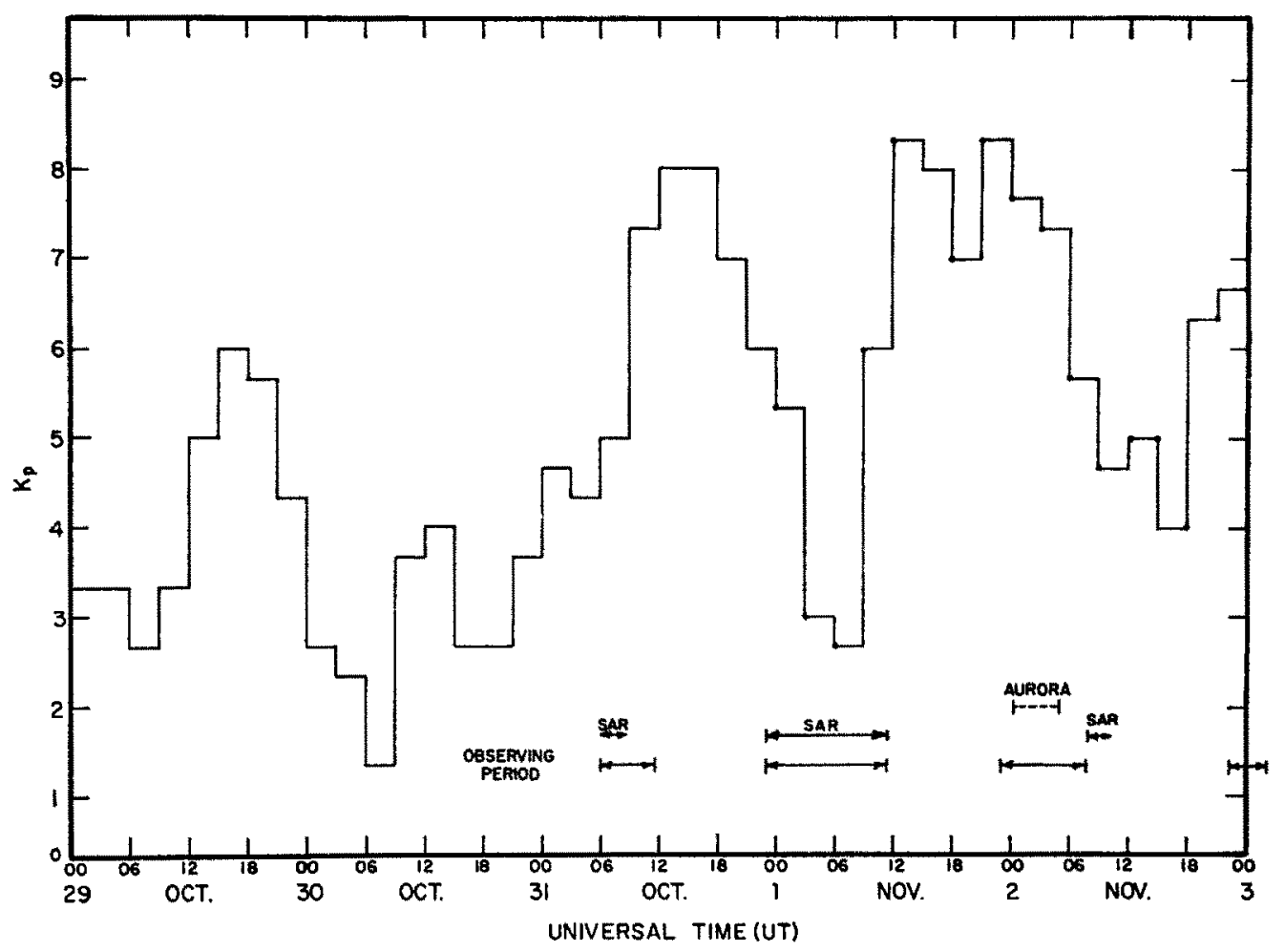

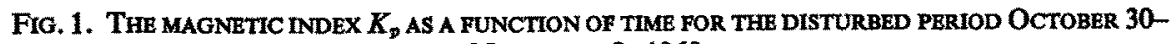
NOVEMBER 2, 1968.

system was performing a North-South vertical circle scan and the red arc appeared as a distinct enhancement of the $6300 \AA$ emission at $50^{\circ} \mathrm{S}$ which was superimposed upon the normal Van Rhijn intensity variation. At the same time, there was a noticeable increase in the $6300 \AA$ emission line in the North which was probably related to auroral activity. No enhancement, in the red arc region, was evident from either the 5577 or $6100 \AA$ photometer scans. The arc moved slowly southward 5 to 10 deg and persisted until 0715 UT when it disappeared. At 0837 UT the $6300 \AA$ emission again increased in the North; however, the red arc did not reappear. The doppler temperature measurements made during the night are shown in Fig. 2 where they are seen to remain nearly constant throughout the night at $1000^{\circ} \mathrm{K}$. The red arc persisted at a time when the $K_{p}$ index was increasing and disappeared even though $K_{p}$ continued to increase during the night.

\section{THE STABLE AURORAL RED ARC OF OCTOBER 31/NOVEMBER 1, 1968}

The magnetic storm which started on the night of October 30/31, 1968, continued during the next day, reaching a $K_{p}$ value of 8 near $1200 \mathrm{UT}$ on October 31 , as shown in Fig. 1 . The storm then decreased in intensity and a red arc was detected south of Ann Arbor shortly after sunset and it persisted throughout the night. Figure 3 shows the position of the peak intensity in the meridian plane from the airglow observatory. At 0030 UT the arc was clearly defined to be at a zenith angle of $15^{\circ}$ south in the meridian plane. Between 0000 and $0300 \mathrm{UT}$ on November 1 , the red arc moved southward with a speed of about $40 \mathrm{~m} \mathrm{sec}^{-1}$, when the speed is calculated at $400 \mathrm{~km}$, the assumed red arc height. The arc 
MICHIGAN AIRGLOW OBSERVATORY

ANN ARBOR, MICHIGAN

DOPPLER TEMPERATURE DURING THE

MAGNETIC STORM OF OCT. 30-NOV.1, 1968.

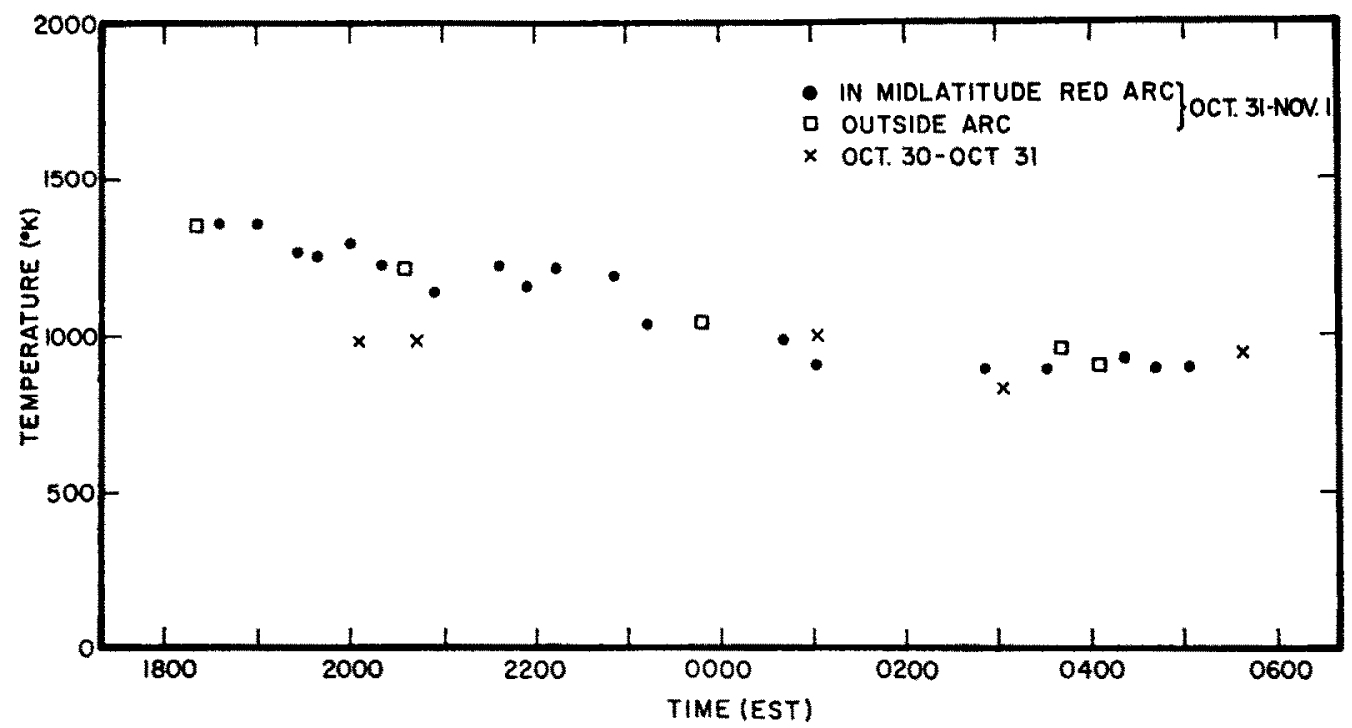

Fig. 2. 6300 Å DOPPLER TEMPERATURE MEASUREMENTS DURING THE MAGNETIC STORM OF OCTOBER 30NOVEMBER $1,1968$.

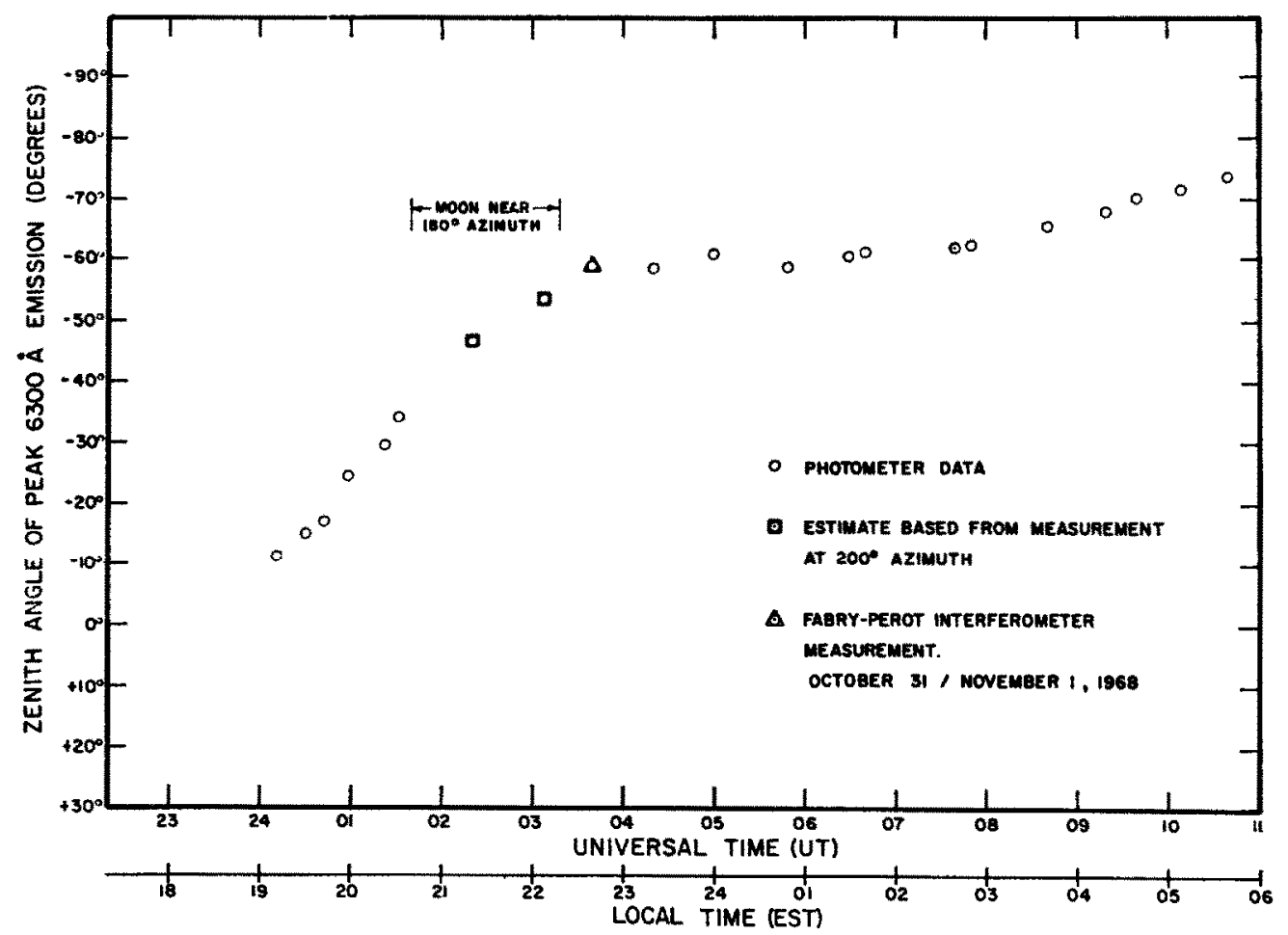

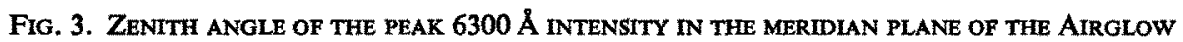
OBSERVATORY AS A FUNCTION OF TIME DURING THE NIGHT OF OCTOBER 31-NOVEMBER 1, 1968. 
settled at a zenith angle near $60^{\circ} \mathrm{S}$ and remained at that position until $0800 \mathrm{UT}$ at which time the arc began moving southward again at a speed of approximately $40 \mathrm{~m} \mathrm{sec}-1$.

Vertical circle surveys were performed with the photometer after the initial location of the red-arc in order to define the horizontal position and extent of the arc. The mirror scanning system was first positioned in azimuth and maintained at that position while a vertical circle scan was performed; going from $+80^{\circ}$ zenith angle in the North through the zenith to $-80^{\circ}$ zenith angle in the South. The mirror was then positioned to a new azimuth angle, usually $20^{\circ}$ from the previous position, and the scan was repeated. Typical scan sequences using the 6300,6100 and $5577 \AA$ filters in the airglow photometer are shown in Figs. 4, 5 and 6. The red-arc appears on the $6300 \AA$ results as a distinct enhancement, superimposed on the normal Van Rhijn intensity increase near the horizon.

The sky conditions during the entire night of October 31/November 1 were generally clear but with some Autumn haze which persisted throughout the night. In addition, a near full Moon was present in the southern sky until about 0730 UT. The Moon's presence is evident in Figs. 5 and 6 which show the 6100 and $5577 \AA$ photometer scans respectively. The increased intensity in the southern sky is caused by the Moon; however, the sharp intensity enhancement evident in the $6300 \AA$ filter scan is not present at the other wavelengths indicating that the arc is a region of predominantly $6300 \AA$ enhancement. Scans of this type, using the $5400,5577,6100$ and $6300 \AA$ filters in the turret photometer, were made throughout the night and only the $6300 \AA$ scans showed the characteristic enhancement, revealing the presence of a red-arc.

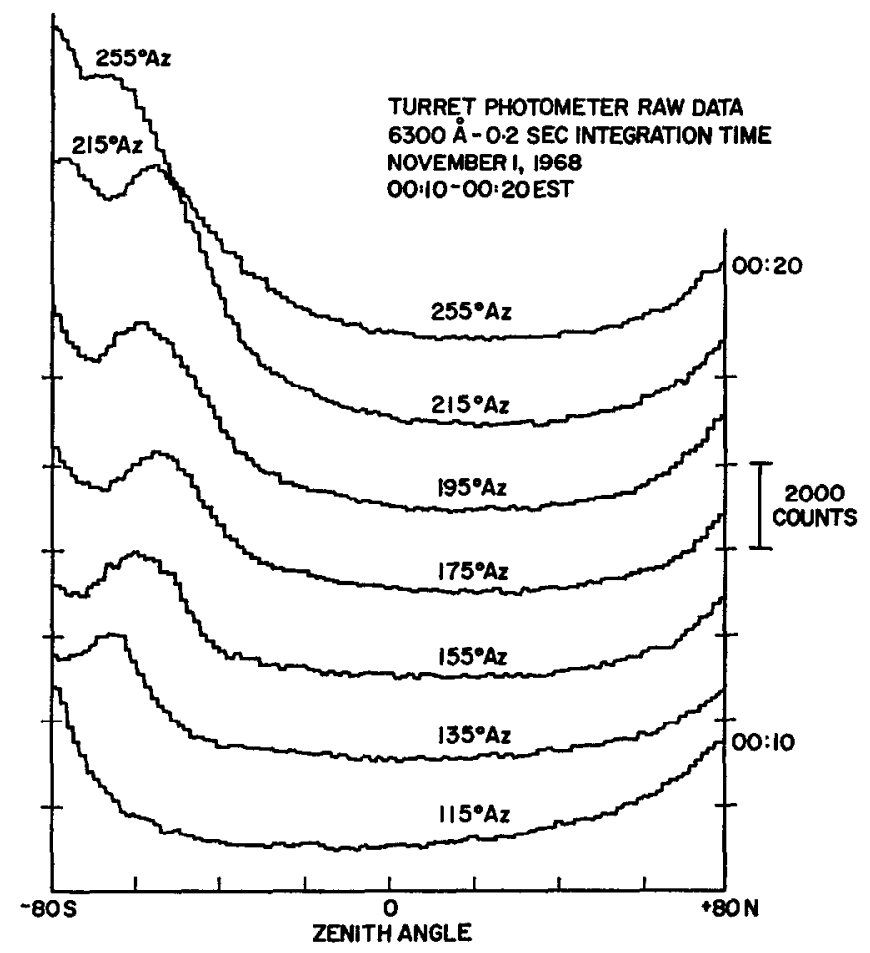

FIG. 4. $6300 \AA$ VERTICAL CIRCLE SCANS WITH THE TURRET PHOTOMETER AT 0010-0020 EST ON NOVEMBER 1, 1968. 




Fig. 5. 6100 $\AA$ VERTICAL CTRCLE SCANS WITH THE TURRET PHOTOMETRR AT 0025-0035 EST ON NOVEMBER 1, 1968.

The Fabry-Perot interferometer was primarily used for Doppler temperature measurements; however these measurements were, at times, interrupted in order to use the interferometer as an extremely narrow interference filter and perform a zenith scan. The interferometer was positioned and maintained on the $6300 \AA$ fringe peak while the mirror scanning system operated in a North-South vertical circle scan crossing the red arc region. The results of the scan are shown in Fig. 8, where the enhanced $6300 \AA$ emission of the SAR-arc clearly stands out. After the scan was completed, the interferometer was moved $0 \cdot 1 \AA$ off of the fringe peak, beyond the wings of the doppler emission line, in order to measure the nearby background. A vertical scan of the interferometer in this position revealed no noticeable enhancement of the background near $6300 \AA$ where the SAR-arc was present. The same measurement was also performed using the $5577 \AA$ green line filter in the interferometer and the results do not show a characteristic enhancement in the arc region indicating that the major emission within the arc is the $6300 \AA$ radiation of atomic oxygen.

The horizontal extent of the red-arc relative to the airglow observatory at Ann Arbor was determined from these figures and the results are shown in Fig. 7, where the peak $6300 \AA$ intensity position is located on an auroral plotting map at various times. In order to position the arc on the map, the height of the peak emission was assumed to be $400 \mathrm{~km}$ which is consistent with the theoretical calculations of Walker and Rees (1968) and Roble (1969) and the results of numerous observations (Roach and Roach, 1963; Rees, 1963; Hoch, Marovich and Clark, 1968). 


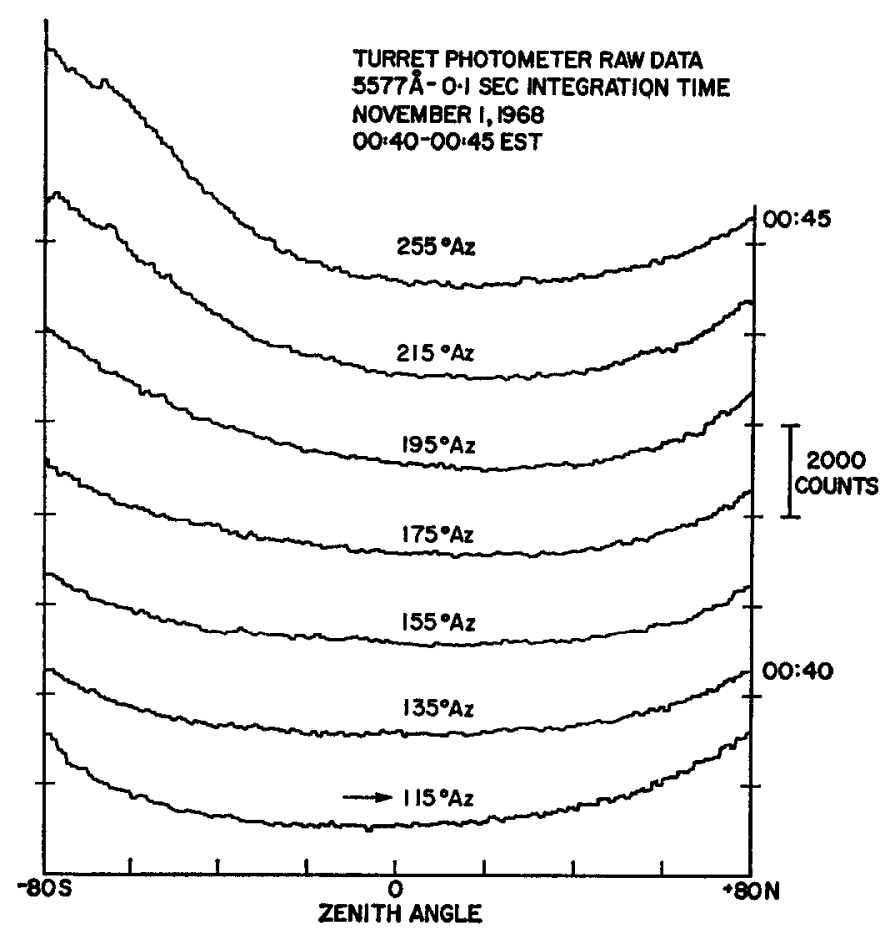

Fig. 6. $5577 \AA$ VERTICAL CIRCLE SCANS WITH THE TURRET PIOTOMETRR AT 0040-0045 EST ON NOVEMBER 1, 1968.

The red arc is seen to lie approximately along constant $L$-shells, although there appears to be a slight departure from true alignment. This result is in agreement with observations reported by Hoch, Marovich and Clark (1968). The red-arc was observed to extend across the eastern U.S., and exhibited a general southward motion throughout the night. The southward drift was associated with both increasing and decreasing $K_{p}$. The red arc position, as determined by Marovich (private communication) from Fritz Peak at 0950 UT, is shown as a dotted line, and when it is considered together with the arc positions determined by the Michigan Airglow Observatory at Ann Arbor, the red-arc is seen to extend across the entire U.S.

The intensity of the arc varied throughout the night, ranging from 100 to $600 \mathrm{R}$ and there also appears to have been some intensity variations along the length of the arc. The intensity was about $300 \mathrm{R}$ at $0130 \mathrm{UT}$ and increased until it reached a maximum of about $600 \mathrm{R}$ near $0515 \mathrm{UT}$. The intensity then dec, eased to $300 \mathrm{R}$ at $0830 \mathrm{UT}$ and shortly before dawn at $0945 \mathrm{UT}$ it was about $100 \mathrm{R}$ or less and the arc was low on the southern horizon. It is not clear to what extent the signal was attenuated by the haze conditions existing near the southern horizon. In the early part of the evening there is also evidence of an enhancement in the North which is probably due to auroral background, although no visual sighting of an aurora was made during the night. The general airglow background appeared to have been slightly enhanced from normal conditions in the early evening but it decayed to about $100 \mathrm{R}$ at $0515 \mathrm{UT}$ and then remained nearly constant for the remainder of the night. 




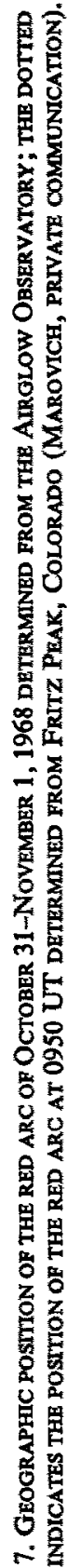
总星 

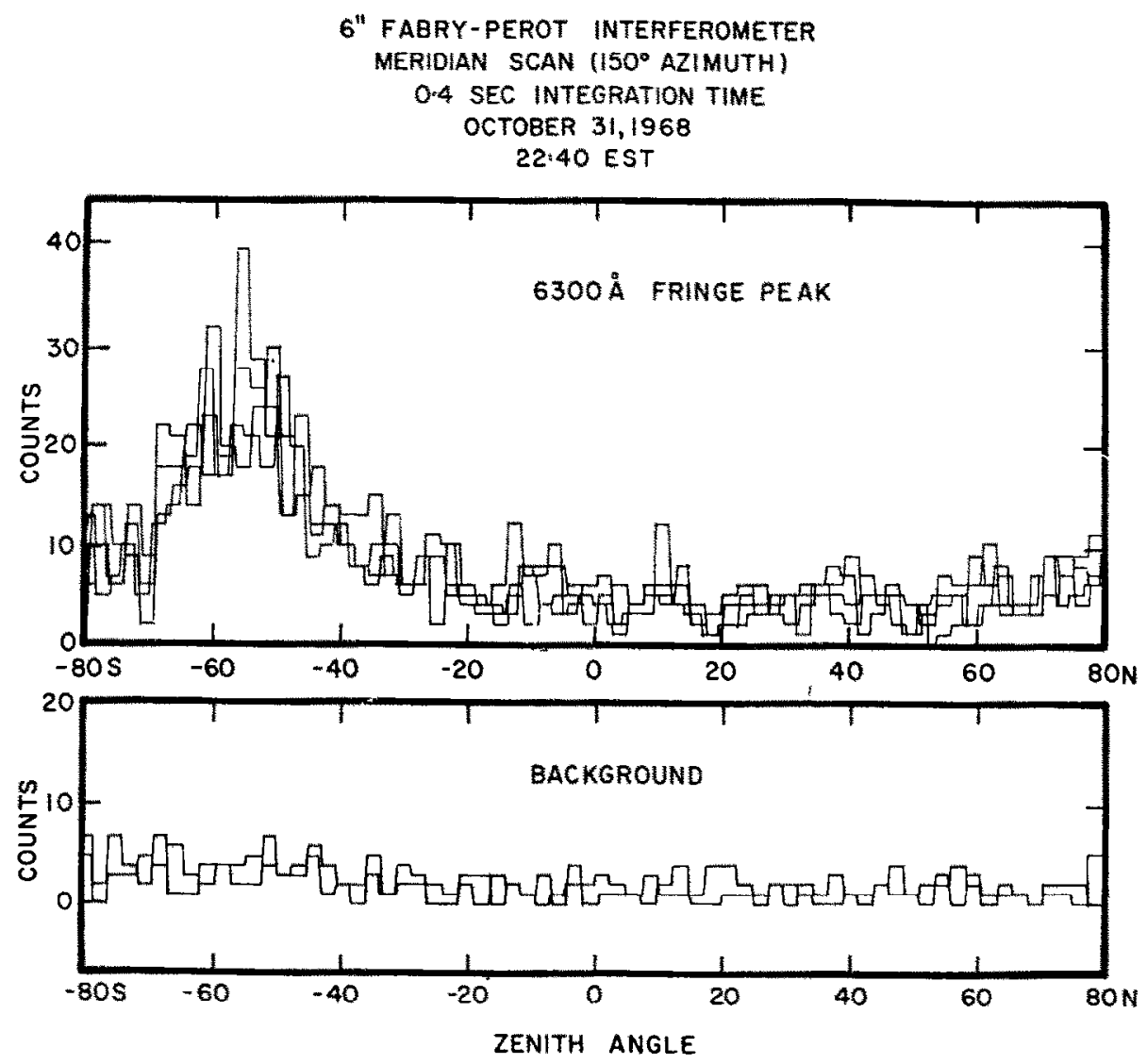

Fig. 8. Fabry-Perot interferometer Vertical Circle sCaN at 0340 UT, October 31, 1968.

The Doppler temperatures within and outside of the arc region and the exospheric temperature variation during the magnetic storm period were measured with the FabryPerot interferometer (Hays, Nagy and Roble, 1969). The doppler temperatures measured during several nights of this magnetic storm were in general agreement with the model predictions of the exospheric temperature (Jacchia, 1965). However, both the amplitude and phase of the doppler temperatures indicated that the exospheric temperature responds more rapidly than the drag results suggest. The Doppler temperature measurements inside and outside of the arc region (Fig. 2) showed no detectable temperature variation, indicating that the neutral gas temperature increase within an arc is negligible. This result is in agreement with the theoretical studies of Roble (1969) on the local heating and subsequent dynamic response of the atmosphere, which showed that the neutral atmosphere reacts to prevent the development of a significant steady state local temperature anomaly in a red arc. Walker and Rees (1968) have shown that if electric fields are the cause of red arcs, the $F_{2}$ ion-temperatures would be in excess of $5000^{\circ} \mathrm{K}$. Such high ion temperatures would effect the neutral gas temperatures, therefore lack of Doppler temperature increase inside the arc could be considered as an indirect evidence against the electric field hypothesis. 


\section{SUMMARY}

The results of the observations described in this paper are summarized below:

(1) Red arcs were observed to be present during two consecutive nights of a magnetic storm period $\left(3<K_{p}<8\right.$ ).

(2) The arc of October 31-November 1 was observed across the entire U.S. The arc was approximately aligned with a constant $L$-shell, although a slight departure from true alignment was apparent; this may indicate a distortion of the $L$-shells during a magnetic storm.

(3) The intensity of the arc varied throughout the night between 100 and $600 \mathrm{R}$. The predominant emission was $6300 \AA$, and no $5577 \AA$ enhancement was apparent within the arc.

(4) The arc of October 31-November 1 moved southward with a velocity of about $40 \mathrm{~m} \mathrm{sec}^{-1}$ during the early night and late morning hours but appeared to be stationary during the middle of the night.

(5) The Doppler temperature observations indicated no temperature increase inside the arc in agreement with theoretical predictions. This result is also an indirect indication that thermal conduction from the protonosphere is the likely source of red arc excitation (Cole, 1965).

Acknowledgements-The authors wish to express their thanks to Dr. M. Gadsden, Mr. E. Marovich and Professor J. Noxon for many helpful suggestions and advice. Numerous members of the High Altitude Engineering Laboratory and the Space Physics Research Laboratory of the University of Michigan were instrumental in designing, constructing and operating the observatory. The research reported here was supported by the Atmospheric Sciences Section, National Scierce Foundation, NSF Grant GA-1025.

\section{REFERENCES}

BARBIER, D. (1954). L'activite aurorale aux basses latitudes, Annls Géophys. 14, 334.

CoLE, K. D. (1965). Stable auroral red arcs, sinks for energy of $D_{t t}$ main phase. J. geophys. Res. $70,1689$.

HAYS, P. B., NAGY, A. F. and RoBLe, R. G. (1969). Interferometric measurements of the $6300 \mathrm{~A}$ doppler temperature during a magnetic storm. J. geophys. Res. 74, 4162.

Hoch, R. J., MAROVICH, E. and ClaRK, K. C. (1968). Reappearance of a stable auroral red arc at midlatitudes. J. geophys. Res. 73, 4213.

ICHAHAWA, T, and KIM, J. S. (1969). Observations of $\mathrm{M}$ arc at Moscow, Idaho, U.S.A. J. atmos. terr. Phys. 31, 547.

JACCHA, L. G. (1965). Static diffusion models of the upper atmosphere with empirical temperature profiles. Smithson. Contrib. Astrophys, 8, 1.

MAROVICH, E. (1966). Fritz peak observations of stable auroral red arcs, summary 1955-1965. ESSA Tech. Rep. No. IER 16-ITSA 16, 68 pp.

PURDY, C. M., MrGill, L. R. and RoACH, F. E. (1961). A new airglow photometer. J. Res. NBS 65C 4, 213.

RRES, M. H. (1963). A method for determining the height and geographical position of an auroral arc from one observing station. J. geophys. Res. 68, 175.

ROACH, F. E. and ROACH, J. R. (1963). Stable $6300 \AA$ auroral arcs in mid-latitudes. Planet. Space Sci. 11, 523.

RoBLe, R. G. (1969). A theoretical and experimental study of the stable mid-latitude red arc (SAR-arc) Ph.D. thesis, Univ. of Michigan.

WALKER, J. C. G. and REEs, M. H. (1968). Excitation of stable auroral red arcs at sub-auroral latitudes. Planet. Space Sci. 16, 915. 